\title{
Evaluación Auténtica y Evaluación Orientada al Aprendizaje en Educación Superior. Una Revisión en Bases de Datos Internacionales
}

\author{
Authentic Assessment and Learning Oriented Assessment in \\ Higher Education. A Review in International Databases
}

\author{
Emilio José Barrientos-Hernán ${ }^{1}$ \\ Víctor M. López-Pastor * 2 \\ Darío Pérez-Brunicardi ${ }^{2}$ \\ ${ }^{1}$ Colegio Público Patriarca Eijo Garay, España \\ ${ }^{2}$ Universidad de Valladolid, España
}

\begin{abstract}
El objetivo de este estudio es realizar un análisis sobre el actual estado de la cuestión en la literatura internacional sobre la temática de la "Evaluación Orientada al Aprendizaje" (Learning Oriented Assessment, LOA) y la "Evaluación Auténtica" (Authentic Assessment, AA) en Educación Superior (HE). La necesidad surge de los nuevos retos que implica el Espacio Europeo de Educación Superior (EEES) en relación con la Evaluación Formativa y sus distintas formas de entenderla. Para ello se ha llevado a cabo una búsqueda bibliográfica en 3 bases de datos internacionales: WOS, ERIC y Google Scholar. Se ha realizado un análisis de contenido a través de un sistema con dos grandes categorías: LOA y AA. Los resultados principales del LOA son: (1) las tareas de evaluación deberían ser diseñadas para estimular el aprendizaje de los alumnos; (2) hay que implicar a los alumnos en la evaluación; (3) el feedback ha de darse en el momento adecuado y debe ser útil para el aprendizaje actual y futuro. La mayoría de experiencias indican aspectos positivos en la utilización de LOA-HE. Los resultados de AA indican que los principales criterios para su definición serían: (1) llevar a cabo tareas auténticas en el aula, con características similares o iguales a las de la vida real; (2) contexto similar o mimetizado al que se encontrarán en el trabajo. La mayoría de experiencias indican que el alumnado valora muy positivamente este tipo de evaluación, por su conexión con la vida real y/o el futuro ambiente de trabajo.
\end{abstract}

Palabras clave: Evaluación auténtica; Evaluación orientada al aprendizaje; Educación superior; Evaluación formativa; Implicación del alumnado en la evaluación.

This paper analyses a review of the international literature on "Learning Oriented Assessment" (LOA) and "Authentic Assessment" (AA) in Higher Education (HE). The European Higher Education Area (EHEA) implies new challenges on Formative Assessmet (FA) and the approaches related to it. A literature review has been carried out using three international databases: WOS, ERIC and Google Scholar. A content analysis has been carried out establishing two categories: LOA and AA. The main results on LOA are: (1) Assessment tasks should be designed to stimulate sound learning practices amongst students; (2) Assessment should involve students actively in engaging with criteria, quality, their own and/or peers' performance; (3) Feedback should be timely and forward-looking so as to support current and future student learning. Most of the experiences show positive aspects using LOA-HE. The results on AA-HE show the main criteria to define it as: (1) do authentic tasks in the classroom with the same or similar characteristics to real life work-tasks; (2) use mimetic or similar contexts to real-work spaces. Most of the experiences show that students assessed very positively this type of assessment due to their connection with real life and/or future world contexts.

Keywords: Authentic assessment; Learning oriented assessment; Higher education; Formative assessment; Student involvement in assessment.

\section{*Contacto: vlopez@mpc.uva.es}

issn: 1989-0397

www.rinace.net/riee/

https://revistas.uam.es/riee
Recibido: 31 de diciembre de 2019

$1^{\text {a }}$ Evaluación: 16 de marzo de 2020

$2^{\text {a }}$ Evaluación: 22 de marzo de 2020

Aceptado: $\quad 31$ de marzo de 2020 


\section{Introducción}

El Espacio Europeo de Educación Superior (EEES) ha conllevado una serie de adaptaciones en las universidades europeas, cambios legislativos y modificaciones que han repercutido en los roles que juegan profesores, alumnos e instituciones (Bretones, 2008; Pérez y otros, 2008); sus principales principios rectores serían: la renovación de la metodología de enseñanza, la adopción del modelo de enseñanza centrado en el estudiante y la adopción del modelo de enseñanza basado en competencias y la promoción del aprendizaje.

Previamente a la introducción del EEES, un gran número de profesores entendían que la única obligación docente era el ser meros transmisores de conocimientos a sus alumnos; y la manera más habitual de evaluar esos conocimientos era a través de un examen final, que se pudiera traducir en una calificación de la asignatura (Palacios y López-Pastor, 2013). Por ello, algunos autores indicaban que las universidades deberían pasar de una cultura evaluativa basada en el examen a otra centrada en una evaluación para el aprendizaje (Dochy, Segers y Dierick, 2002). En este sentido, el EEES parece implicar que los profesores modifiquen los sistemas de evaluación-calificación tradicionales, dado que la actividad evaluadora ha sido considerada como una de las grandes carencias en las aulas y una de las competencias docentes que más debe cambiar (Zabalza, 2003). Sin embargo, algunas investigaciones recientes muestran cambios en las facultades españolas encargadas de la formación inicial del profesorado (FIP), sobre todo desde el año 2010, donde un tercio del profesorado ya empieza a utilizar sistemas de Evaluación Formativa y Compartida (EFyC) en FIP (Palacios y López-Pastor, 2013). En cambio, otros autores se muestran críticos con este proceso; Bretones (2008) consideraba que las repercusiones de los cambios que se esperaban por la introducción del EESS en España habían sido muy leves y, en la mayoría de los casos, simplemente a nivel burocrático.

Según Palacios y López-Pastor (2013), el sistema de evaluación formativo es de los que mejor se adecua al EESS, ya que su principal finalidad es mejorar el aprendizaje del alumnado y el funcionamiento del proceso de enseñanza y aprendizaje (López-Pastor, 2008 y Pérez y colaboradores, 2008). Por lo tanto, estaría dando respuesta a cómo valorar el proceso del alumno y, a la vez, tener en cuenta el aprendizaje alcanzado por los mismos. No existen excesivos estudios bibliográficos relacionados con la "evaluación formativa en educación superior" (EF en HE) y los realizados son bastante antiguos. Uno de los de mayor impacto fue llevado a cabo por Black y Wiliam (1998), sobre el término: "Formative Assessment (FA)". Para ello recogieron un total de 681 publicaciones relacionadas con la aplicación de un sistema de FA en los colegios y universidades. Entre otros aspectos, resaltaba la reconceptualización del término FA, debido a la confusión que estaba generando, ya que muchas de las publicaciones afirmaban llevar a cabo un sistema de FA pero en la práctica eran similares al sistema de evaluación sumativo. Posteriormente Gaunlett (2007) llevó a cabo una búsqueda bibliográfica del concepto de "FA en Higher Education (FA-HE)" entre 1998-2007, seleccionando 46 estudios. Este autor afirma que para que se produzca una evaluación que se pueda definir como formativa hay que considerar el tipo de feedback que se realiza, siendo el feedback formativo el elemento clave.

En España, la mayoría de los estudios relacionados con el término de FA-HE comenzaron en el año 2000, pero el mayor incremento ha sido a partir de 2009 (López-Pastor y Sicilia 2017). Barrientos (2013) realiza una búsqueda bibliográfica internacional del concepto de FA-HE entre 2007-2013, así como de otros términos relacionados con el anterior, como: 
“Alternative Assessment"-ALA (Evaluación Alternativa), "Authentic Assessment"-AA (Evaluación Auténtica), “Assessment For Learning"-AFL (Evaluación Para el Aprendizaje), "Learning Oriented Assessment"-LOA (Evaluación Orientada al Aprendizaje), "Electronic Assessment"-EA (Evaluación Electrónica), "Students Involvement"-SI: Peer-Assessment, SelfAssessment y Collaborative Assessment (Implicación del alumnado en su evaluación: evaluación entre iguales, autoevaluación y evaluación colaborativa). Revisando la literatura internacional especializada puede comprobarse que el término $\mathrm{FA}$ ha evolucionado hacia otros más centrados en el proceso de aprendizaje del alumnado, como: ALA, AFL, LOA o AA (Carless, 2007; Lorente y Kirk, 2013; López-Pastor y Sicilia, 2017; López-Pastor, Kirk, Lorente-Catalan, Macphail y MacDonald, 2013).

Dos de los conceptos más interesantes de esta red nomológica son los de LOA y AA. De ellos no existen prácticamente estudios y están estrechamente relacionados con el sistema de FA (López-Pastor y Sicilia, 2017). López-Pastor y otros (2013) consideran la AA como un sistema de evaluación estrechamente relacionado con la FA. El concepto de AA hace referencia al hecho de que las técnicas, instrumentos y actividades de evaluación estén claramente aplicados en situaciones, actividades y contenidos reales del aprendizaje. Se opone, por tanto, a las situaciones puntuales y artificiales de evaluación, alejadas de la práctica real o de la aplicación real de dichos conocimientos (Archbald 1991; Archbald y Newman, 1988; Gulikers y otros, 2004; Rule, 2006). El término LOA es utilizado por diferentes autores (Boud y Falchikov, 2006; Carless, 2007; Carmona y Flores, 2008; Ibarra, Rodríguez y Gómez, 2012; Keppell y Carless, 2006) y tiene tres características: (1) el proceso de evaluación debe implicar activamente al estudiante como evaluador; (2) se debe aportar retroalimentación y proalimentación; y (3) las tareas de evaluación deben ser tareas auténticas, ligadas con la realidad profesional (Carless, 2007; Carless, Joughin y Mok, 2006; Ibarra, Rodríguez y Gómez, 2012; Quesada, Rodríguez e Ibarra, 2013).

Según Gessa (2011) el EESS implica un sistema de evaluación en el que se promueva una LOA en el que predominen tareas de carácter auténtico con los alumnos, siendo LOA y AA los dos sistemas que mejor encajarían en esa descripción. Por su parte, Bretones (2008) señala que en España se ha publicado poco sobre LOA y AA en HE.

Debido a la escasez de revisiones bibliográficas realizadas sobre esos conceptos, consideramos interesante y necesario realizar un estudio a fondo del estado de la cuestión de ambos. Por ello, el presente estudio tiene como objetivos:

1. Realizar una búsqueda bibliográfica en tres bases de datos internacionales de los conceptos: "Learning Oriented Assessment in Higher Education (LOA-HE)" y "Authentic Assessment in Higher Education (AA-HE)".

2. Analizar el estado de la cuestión de los términos anteriores en la literatura internacional especializada.

\section{Metodología}

Se ha llevado a cabo una búsqueda bibliográfica en tres bases de datos internacionales: "WOS", "Google Scholar" y "ERIC". Tras la realización de las correspondientes búsquedas y selección de documentos se procedió a realizar un análisis bibliográfico y de contenido de los mismos. Los conceptos de búsqueda han sido: LOA-HE y AA-HE. 
Somos conscientes de que dichas búsquedas no agotan todo lo publicado al respecto, porque al limitarnos a estas bases de datos pueden quedarse fuera otros tipos de documentos, como libros y aportaciones a congresos. Se trata de una limitación a asumir en cualquier búsqueda de este tipo.

\subsection{Técnica e instrumentos de recogida de datos}

En cada una de las bases de datos se ha realizado la búsqueda con unos ajustes distintos, ya que cada uno de los buscadores dispone de unos criterios de búsqueda diferentes, siendo imposible realizar la búsqueda con los mismos parámetros en todos los buscadores. El procedimiento fue el siguiente: (a) en Google Scholar se ha realizado la búsqueda con los parámetros: "palabras exactas dentro del artículo"; (b) en ERIC se ha analizado: "topic" y "title"; y (c) en WOS, se ha realizado por: "topic" y "title". Por ejemplo, al buscar en Google Scholar el concepto de "AA-HE”, hemos recogido información de todos aquellos artículos que muestren dentro del documento ese concepto concreto. En cambio, en ERIC y WOS utilizo como "title": "AA" y como "topic": "HE".

Del total de publicaciones encontradas, hemos seleccionado aquellas que aportan suficiente información como para poder categorizarlas después. Si la misma publicación aparece en varias bases de datos sólo se selecciona en una de ellas. Se ha realizado la búsqueda con fecha de inicio abierta y hasta mayo de 2018 .

Para llevar a cabo la recogida de datos se ha utilizado el cuadro 1, en la que se vuelcan las publicaciones encontradas y seleccionadas en la búsqueda realizada en las tres bases de datos citadas. Para la selección de documentos se han seguido dos criterios: (1) que se cite dentro del documento la frase entera objeto de búsqueda: LOA-HE o AA-HE; y (2) que aporte información relevante de los conceptos de búsqueda: conceptualización, características, clasificaciones, experiencias y/o resultados.

Cuadro 1. Resultados de las búsquedas bibliográficas realizadas

\begin{tabular}{lccc}
\hline \multicolumn{1}{c}{ CONCEPTO } & BASE DE DATOS & RESULTADOS & SELECCIÓN \\
\hline \multirow{2}{*}{ Learning Oriented Assessment in } & WOS & 16 & 3 \\
Higher Education & ERIC & 12 & 3 \\
& Google Scholar & 18 & 6 \\
\hline \multirow{2}{*}{ Authentic Assessment in Higher } & WOS & 34 & 7 \\
Education & ERIC & 68 & 8 \\
& Google Scholar & 59 & 13 \\
\hline
\end{tabular}

Fuente: Elaboración propia.

\subsection{Técnica de análisis de datos}

Para elaborar el informe de resultados y realizar las conclusiones se ha llevado a cabo un proceso de categorización. En el cuadro 2 se han clasificado cada una de las categorías y subcategorías que se han establecido, todas ellas con el objetivo de estructurar y organizar la información recogida.

Cuadro 2. Categorías y subcategorías para analizar los documentos seleccionados

\begin{tabular}{ll}
\hline \multicolumn{1}{c}{ CATEGORÍAS } & \multicolumn{1}{c}{ SUBCATEGORÍAS } \\
\hline \multirow{3}{*}{ 1. LOA-HE } & 1.1- Origen del concepto \\
& 1.2- Criterios para ser LOA \\
& 1.3- Experiencias y resultados de su aplicación \\
& 2.1- Origen del concepto \\
2. AA-HE & 2.2- Criterios para ser AA \\
& 2.3- Experiencias y resultados de su aplicación \\
\hline
\end{tabular}

Fuente: Elaboración propia. 


\section{Resultados}

Los datos están estructurados en base a dos grandes apartados, que obedecen a las categorías y subcategorías establecidas previamente. En las dos categorías se han utilizado cuadros-resumen de los documentos encontrados, ordenando los documentos cronológicamente y aportando una breve explicación del motivo por el que se ha seleccionado ese documento.

\subsection{Learning Oriented Assessment in Higher Education}

De esta categoría se han seleccionado 11 de los 46 documentos encontrados (cuadro 3).

Cuadro 3. Resultados de la búsqueda del concepto: "LOA-HE"

\begin{tabular}{|c|c|}
\hline $\begin{array}{l}\text { AUTORES Y } \\
\text { AÑO }\end{array}$ & SELECCIÓN \\
\hline $\begin{array}{c}\text { Boud y } \\
\text { Falchikov, } 2006\end{array}$ & Se explican ejemplos de prácticas de LOA in HE. \\
\hline $\begin{array}{l}\text { Carless, Joughin } \\
\text { y Mok, } 2006\end{array}$ & $\begin{array}{l}\text { Se centran en una LOA, dejando de lado y no comparándolo con el de FA, ya } \\
\text { que entienden que hay confusión con este término (Yorke, 2003). }\end{array}$ \\
\hline Carless, 2007 & $\begin{array}{l}\text { Introduce el término de LOA y explica sus tres características principales, } \\
\text { reflexiona sobre el proyecto llevado a cabo en Hong Kong sobre LOA y } \\
\text { explica como la LOA puede ser implementada en la práctica. }\end{array}$ \\
\hline Lombard, 2008 & $\begin{array}{l}\text { Desarrollo del pensamiento crítico a través de una LOA. Además, definen } \\
\text { LOA. }\end{array}$ \\
\hline $\begin{array}{l}\text { Carmona y } \\
\text { Flores, } 2008\end{array}$ & $\begin{array}{l}\text { Insisten en la necesidad de que la evaluación se convierta en estrategia para } \\
\text { la mejora de los aprendizajes. Para ello, deben cumplirse tres condiciones: 1) } \\
\text { las tareas de evaluación deben ser también tareas de aprendizaje; 2) se ha de } \\
\text { proporcionar retroalimentación para orientar el trabajo futuro; 3) implicar a } \\
\text { los estudiantes en el proceso de evaluar su propio trabajo. }\end{array}$ \\
\hline Carless, 2009a & $\begin{array}{l}\text { Señala las tres características para ser LOA y analiza las tensiones que } \\
\text { genera una evaluación que cumpla con el: "doble deber" que señala Boud. }\end{array}$ \\
\hline Carless, 2009b & $\begin{array}{l}\text { Uno de los factores que limitan la introducción de prácticas de LOA es la } \\
\text { creencia de la poca confianza en las mismas. El autor explica cómo podría ser } \\
\text { desarrollada esa confianza. }\end{array}$ \\
\hline Carless, 2015a & $\begin{array}{l}\text { Selección de } 5 \text { profesores premiados por sus buenas prácticas educativas y } \\
\text { analizando como sus prácticas evaluativas y su modelo personal de } \\
\text { evaluación orientada al aprendizaje. }\end{array}$ \\
\hline Carless, 2015b & $\begin{array}{l}\text { Propone un modelo de LOA centrada en los tres procesos descritos anteriormente. } \\
\text { Además, analizar: 1) las tareas de evaluación que llevan a cabo los alumnos; 2) } \\
\text { desarrollo de las capacidades evaluativas de los alumnos: 3) El compromiso de los } \\
\text { estudiantes con respecto al feedback. }\end{array}$ \\
\hline $\begin{array}{l}\text { Rodríguez, } \\
\text { Quesada e } \\
\text { Ibarra, } 2016\end{array}$ & $\begin{array}{l}\text { Analiza los efectos de una propuesta de LOA-electrónica llevada a cabo por } \\
\text { profesores universitarios. Valoran diferentes aspectos de la experiencia } \\
\text { implementada. }\end{array}$ \\
\hline $\begin{array}{l}\text { Canabal y } \\
\text { Margalef, } 2017\end{array}$ & $\begin{array}{l}\text { Analiza los procesos de Feedback y algunos instrumentos de evaluación } \\
\text { desde una perspectiva centrada en LOA. }\end{array}$ \\
\hline
\end{tabular}

Fuente: Elaboración propia.

\subsection{Origen del concepto $L O A$}

Según Boud y Falchikov (2006), la LOA es un sistema de evaluación que no se ciñe únicamente al contexto del aula y debe ser útil para los alumnos a la hora de contextualizar lo aprendido en la esfera de la vida real y del trabajo. Por su parte, Carless, Joughin y Mok (2006) señalan la LOA como un concepto no comparable con el de FA, ya que entienden que hay confusión con este término. Por lo que, el término LOA surge para dar respuesta al doble deber que ha de cumplir la evaluación en HE (Boud y Falchikov, 2006), teniendo 
que aunar tanto las características de la evaluación sumativa o certificación del alumno como las de la FA o progreso de aprendizaje del alumnado (Carless, 2007, 2009, 2015a).

\subsection{Criterios para ser $L O A$}

La LOA se caracteriza por tres principios (Carless 2007, 2009a, 2015a; Carless, Joughin y Mok, 2006; Carmona y Flores, 2008): (1) las tareas de evaluación deberían ser diseñadas para estimular el aprendizaje de los alumnos; (2) la evaluación tiene que implicar a los alumnos en su propia evaluación y en la evaluación de otros compañeros; y (3) el feedback o retroalimentación ha de darse en el momento adecuado a los alumnos y que sea útil para el aprendizaje actual y futuro de los mismos. Rodríguez, Quesada y Ibarra (2016) denominan "LOA Electrónica" a una reformulación de los tres principios de LOA, pero concretados en un contexto de evaluación electrónica: (1) llevar a cabo tareas de evaluación electrónica como tareas de aprendizaje; (2) utilizar el e-feedback (retroalimentación electrónica) como feedforward (retroalimentación) que permita avanzar hacia delante, en este caso permitiendo a los alumnos orientar su aprendizaje actual y futuro; y (3) participación de los alumnos en la evaluación electrónica a través de estrategias como autoevaluación, evaluación entre iguales y evaluación colaborativa.

Lombard (2008) le da un cariz diferente a la LOA, focalizada en desarrollar el pensamiento crítico de los alumnos. Además, señalan que LOA puede ser descrita como una sinergia entre instrucción, aprendizaje, evaluación y feedback aportado durante la clase, la cual de manera consciente intenta ayudar a los aprendices para apoyar y estimular su competencia en pensamiento crítico.

\subsection{Experiencias y resultados de la aplicación LOA}

Carless (2007) explica la primera experiencia en la que se pone en práctica la LOA, en el Instituto de Educación de Hong Kong, a lo largo de cuatro años (2002-2006), en el que se involucró a otras universidades tanto nacionales como extranjeras. El principal objetivo era identificar, promover y divulgar buenas prácticas de LOA-HE. Desde el proyecto se invitó a casi 400 profesores a implementar LOA en sus aulas, de los que 40 realizaron un informe final en el que exponían sus resultados. También realizó un programa de formación inicial con 35 profesores, con una duración de 12 semanas (30 horas). Este programa estaba dirigido a que los profesores conocieran y desarrollaran algunas de las mejores y más innovadoras prácticas docentes realizadas utilizando LOA; que fue valorado de manera muy positiva.

Carless (2015b) realiza una revisión de la bibliografía sobre evaluación en HE, concluyendo en los tres principios de la LOA explicados anteriormente. A partir de estos principios, proponen dos preguntas que han de ser contestadas a lo largo de su estudio: (1) ¿Cómo es la LOA que llevan a cabo una muestra de profesores premiados en sus prácticas docentes?; y (2) ¿Cuáles son las principales percepciones de profesores y alumnos sobre el uso de la LOA? En los resultados hablan de casos concretos: por ejemplo, en el caso de un profesor de arquitectura, son varios los factores que mejoran y facilitan los procesos evaluativos: el uso del portafolio como actividad de evaluación, los procesos de autoevaluación generados en la revisión de los trabajos de los estudiantes y el feedback dialógico creado durante todo el proceso en, las interacciones entre profesores y estudiantes. En otros casos, las clases de historia y derecho eran muy numerosas, pero ello no representó una barrera a la hora de llevar a cabo prácticas de LOA; por lo que los autores consideran que estas experiencias muestran que la determinación y compromiso 
de los profesores puede derribar algunos de los problemas que puedan surgir para desarrollar procesos de evaluación efectivos.

Rodríguez y otros (2016) analizan los efectos de la puesta en práctica de un programa de formación dirigido a profesores universitarios de "LOA electrónica". Los resultados muestran que el profesorado que usa herramientas electrónicas de LOA mejora la competencia y habilidad en evaluación, mencionando que se sienten más preparados para llevar a cabo procesos de LOA electrónica en sus prácticas docentes, tienen más recursos bibliográficos, conocen fuentes de información relacionadas con LOA y son capaces de utilizar y poner en práctica procesos de evaluación colaborativa y participativa en sus prácticas docentes.

Canabal y Margalef (2017) llevan a cabo un análisis de los procesos de feedback que se generan entre estudiantes y profesores desde una perspectiva de LOA. El contexto de la investigación es el Máster en Docencia Universitaria, en el que participaron profesores de distintas disciplinas de la universidad. Los participantes señalaron que el proceso de elaboración de cartas personales como instrumentos de feedback aumentó la motivación y aprendizaje de los participantes. Además, en este estudio se clasificaron y analizaron las condiciones en la que los procesos de feedback tienen mayor impacto en una LOA.

Sin embargo, hay varios documentos que citan algunas dificultades para la introducción de sistemas LOA. Carless (2009a) señala que el doble deber que tiene que cumplir la evaluación ha generado una serie de tensiones entre profesores y alumnos, proponiendo una serie de estrategias de evaluación que los estudios han indicado como efectivas, entre las que se encuentran el uso del portafolio (portfolio) y de trozos de texto con las claves de los aspectos realizados (patchwork texts). Por otro lado, Carless (2009b) analiza como la desconfianza es uno de los factores que incide para en la no utilización de sistemas de evaluación alternativos y LOA. Explica cómo ha de ser desarrollada la confianza, algunas de las barreras que hay que eliminar y la relación entre confianza y buenas prácticas evaluativas. Algunas de las estrategias que propone para crear confianza son: (1) sistemas de evaluación que puedan ser justificados de manera teórica y práctica, que además aporten la seguridad para defender nuestras prácticas evaluativas frente a evaluadores internos y externos; y (2) gran transparencia en los procesos de evaluación.

Los principales resultados de esta primera categoría podrían ser los siguientes: (1) Tanto el origen, los criterios y la mayoría de los trabajos sobre la LOA parecen estar ligados al autor Carless (2006, 2007, 2009b, 2015), aunque también aparecen otros autores que aportan trabajos e ideas novedosas en algunas temáticas; y (2) los resultados parecen ser positivos en la mayoría de los estudios realizados aunque también surgen problemas y resistencias, como en cualquier tipo de innovación educativa. Alguno de los estudios aporta algunas posibles estrategias para minimizar o solucionar dichas dificultades.

\subsection{Authentic Assessment in HigherEducation}

El segundo concepto de análisis es el de AA in HE; se han seleccionado 28 de los 161 documentos encontrados (cuadro 4).

Cuadro 4. Resultados de la búsqueda del concepto: "AA-HE"

\begin{tabular}{|c|c|}
\hline AUTORES Y AÑO & SELECCIÓN \\
\hline $\begin{array}{l}\text { Archbald y Newman, } \\
1988\end{array}$ & $\begin{array}{l}\text { Origen del término AA del que parten la mayoría de estudios } \\
\text { posteriores. }\end{array}$ \\
\hline Archbald, 1991 & $\begin{array}{l}\text { Explica los principios, experiencias y temas relacionados con AA- } \\
\text { HE, todo ello relacionado con el mundo del trabajo }\end{array}$ \\
\hline
\end{tabular}


Cumming y Maxwell, 1999

Gulikers, Bastiaens y Kirshner, 2004

Rule, 2006

Gulikers, Kester, Kirschner y Bastiaens, 2008

Keyser y Howell, 2008 Hassanpour, Utaberta, Abdullah y Tahir, 2011 $\mathrm{Vu}, 2011$

Bohemia y Davison, 2012

Eddy y Lawrence, 2013

Bosco y Ferns, 2014

Ashford-Rowe, Herrington y Brown, 2014

Biddle, 2014

Gonzalez-DeHass y

Willems, 2015

Heinzen, Landrum, Gurung y Dunn, 2015

Latorre y Varela, 2015

Kearney, Perkins y

Kennedy-Clark, 2016

Sullivan y McConnell, 2017

Prieto, Llácer y Escobar, 2017

Ghosh, Bowles, Ranmuthugala y Brooks, 2017

Febriana y Arlianty, 2017

McDermott y otros, 2017
Concepto relacionado con el mundo del trabajo y clasifican las propiedades de la AA en: desempeño, contexto, complejidad y competencia.

Describen un marco de referencia de la AA con cinco dimensiones para que pueda tener lugar: (1) la tarea de evaluación; (2) el contexto físico de la tarea; (3) el contexto social de la tarea; (4) la evaluación de resultados; (5) los criterios de evaluación.

Revisión de artículos de AA-HE, aunando en cuatro las características de las que se deben componer las actividades de AA: (1) involucrar problemas del mundo real que mimeticen situaciones de trabajo de los profesionales de esa materia; (2) incluir situaciones con respuestas abiertas y múltiples opciones, habilidades de reflexión y metacognición; (3) involucrar a los alumnos en debates y en el aprendizaje de habilidades sociales; (4) permitir a los estudiantes dirigir su propio aprendizaje.

Estudio en el que analizan cómo los estudiantes perciben la AA y cómo influye en sus aprendizajes.

Realizan una revisión de artículos relacionados con AA-HE. AA como una evaluación diferente a la tradicional o sumativa, dándole importancia no sólo a adquirir conocimientos. Relaciona la AA con el mundo de la educación formal. Relación de AA con el mundo del trabajo Aportan cuatro características de las que se tiene que componer la AA: (1) la evaluación es un proceso y no es algo estático y puntual; (2) la AA supone evaluar aprendizajes experimentales; (3) que sean varias las personas que evalúen el trabajo del estudiante, incluyendo la auto-evaluación o la revisión por una audiencia pública; y (4) la AA tiene que ofreces más oportunidades al aprendiz para decidir en su evaluación. Clasifican las actividades de AA en base a dos criterios: (1) autenticidad; y (2) proximidad.

Realizan una revisión bibliográfica y reformulan en ocho preguntas las características que deben tener las prácticas de AA.

Desde el programa de doctorado de la Universidad Central de Florida llevan a cabo en colegios y distritos varios proyectos relacionados con AA.

Cursos de formación en la facultad de psicología con futuros profesores, en los que utilizan ambientes de aprendizaje auténtico y como llevar a cabo una AA.

Señalan las ocho grandes dificultades encontradas para implementar AA-HE.

Uso de las rúbricas para que aporten feedback y feedforward. Ponen en práctica una AA en la que los alumnos realizan autoevaluaciones y evaluaciones a otros compañeros bastante precisas.

Las universidades deben actualizarse al siglo XXI, entre otras cosas introduciendo una AA.

Utilizan el portafolio como uno de los instrumentos más populares para pasar de una evaluación tradicional a otra basada en AA

Realizan una revisión de estudios de AA, concluyendo que es necesario que estas prácticas cumplan aspectos como fiabilidad y validez.

Desde AA, desarrollan la incidencia de una herramienta que sea capaz de evaluar: conocimientos, actitudes, auto-eficacia y evaluación entre iguales. Facultad de Química en Indonesia. Utilización de una AA que este en consonancia con la metodología que se lleva a cabo. 
Santos, 2017

Murphy, Fox, Freeman y

Hughes, 2017

Kaider, Hains-Wesson y

Young, 2017

Nguyen, 2017

James y Casidy, 2018
Pone en práctica una AA en la Facultad de Farmacia en la que los estudiantes encuentran difícil autoevaluarse o evaluar a otros compañeros,

Aportan una definición de AA y una guía para su implementación en el aula

Clasifican las actividades de AA en base a dos criterios: autenticidad y proximidad.

Estudio en Vietnam en el que los futuros profesores introducen tres tareas de AA en sus prácticas.

Aplican AA en una muestra de 120 alumnos en la Facultad de Negocios con resultados positivos.

Fuente: Elaboración propia.

\subsection{Origen del concepto $A A$}

Archbald (1991) fue uno de los primeros autores que definió el término de AA-HE como una evaluación utilizada para evaluar experiencias y logros del mundo real. Además, señalan que la mejor manera de evaluar el aprendizaje que ocurre en el aula clase es aquella realizada de manera auténtica (Archbald y Newman, 1988; Keyser y Howell, 2008). Cumming y Maxwell (1999) añaden que la aplicación de AA variará dependiendo de las creencias educativas de quién lo lleva a la práctica, indicando que la AA es una forma coherente de llevar a cabo la evaluación, siempre y cuando esté en consonancia los procesos de enseñanza y aprendizaje.

Muchos estudios relacionan el concepto AA con el mundo del trabajo (Archbald, 1991; Archbald y Newman, 1988; Bohemia y Davison, 2012; Cumming y Maxwell, 1999). Vu (2011) añade que la AA implica unos fuertes lazos con el mundo de la educación formal, centrando su propuesta con el mundo educativo, dirigida a promover los aprendizajes de los estudiantes y con un carácter más humanizador y que sirva para preparar a los estudiantes para el futuro; además de evaluar los aprendizajes, el alumnado también interioriza sus conocimientos y aprende como actuar con las personas que tiene alrededor. Murphy y otros (2017) añaden que la AA es un tipo de evaluación en la cual los estudiantes tienen que desarrollar tareas del mundo real en las que demuestren la aplicación de conocimientos esenciales y habilidades clave.

En la misma línea educativa, Hassanpour y otros (2011) definen AA como una evaluación que no está solo centrada en la adquisición de conocimientos como fin último, sino también en evaluar durante el proceso el uso de conocimientos, habilidades y estrategias para la resolución de problemas con múltiples opciones de solución. Además, utilizan la AA como sinónimo de una evaluación alternativa y diferente a la que ellos denominan "estándar o tradicional". En línea con lo anterior, Sullivan y Mcconnell (2017) indican que las facultades universitarias deben proponerse una serie de retos que estén en concordancia con las necesidades y expectativas del estudiante universitario del siglo XXI. Uno de ellos es llevar a cabo una AA entendida como una evaluación alternativa y/o FA que este en coherencia con la metodología utilizada. McDermott y colaboradores (2017) señalan la necesidad de llevar a cabo un tipo de AA que este en concordancia con metodologías como el aprendizaje situado y aprendizaje social, señalando la importancia de una evaluación que guie el aprendizaje.

\subsection{Criterios para ser $\boldsymbol{A} A$}


Cumming y Maxwell (1999) clasificaron la AA y sus propiedades en cuatro subgrupos: desempeño, contexto, complejidad y competencia. Además, asociaron las tres primeras con teorías del aprendizaje: (1)“desempeño”; similitud entre las tareas de evaluación y las de la vida real; (2) "contexto"; los estudiantes suelen transferir muy pocas de las destrezas aprendidas en el aula a situaciones del mundo real; se relaciona con la teoría del aprendizaje situado y la necesidad de utilizar contextos de aprendizaje lo más reales posibles; (3) "complejidad"; los estudiantes están mejor preparados para desarrollar la capacidad de resolución de problemas si el aprendizaje y la evaluación están inherentes en escenarios complejos que simulen situaciones originales y genuinas; (4) "competencia"; actividades que requieren del uso de diferentes habilidades para su desempeño.

Para Gulikers y colaboradores (2004), la AA se distingue por tener cinco dimensiones: (1) la tarea de evaluación; (2) el contexto físico de la tarea; (3) el contexto social de la tarea; (4) la evaluación de resultados; y (5) los criterios de evaluación. Además, sostienen que las tareas llevadas a cabo deben reflejar la competencia que necesita ser evaluada, su contenido tiene que representar situaciones o problemas de la vida de real y los estudiantes deberían llevar a cabo procesos de reflexión similar a los utilizados para solucionar ese mismo de situaciones cuando ocurren en la vida real.

Rule (2006) realiza una revisión de ejemplos de AA en HE y señala cuatro características de las que se deben componer las actividades de AA: (1) involucrar problemas del mundo real que mimeticen situaciones de trabajo de los profesionales de esa materia; (2) incluir situaciones con respuestas abiertas y múltiples opciones, habilidades de reflexión y metacognición; (3) involucrar a los alumnos en debates y en el aprendizaje de habilidades sociales; y (4) permitir a los estudiantes dirigir su propio aprendizaje.

Por otra parte, algunos estudios analizan las actividades de AA en base a dos aspectos (Bosco y Ferns, 2014; Kaider, Hains-Wesson y Young, 2017): (1)“autenticidad”; siendo más genuina cuanto más parecida son las habilidades necesarias para desarrollar la tarea simulada a la que se encontraría en la realidad; y (2)“proximidad"; siendo mayor cuando el contexto reúne las mismas características o similares a las encontradas en la realidad.

Eddy y Lawrence (2013) presentan una estructura conceptual de cuatro fases en AA: (1) la evaluación es un proceso y no es algo estático y puntual; (2) la AA supone evaluar aprendizajes experimentales; (3) que sean varias las personas que evalúen el trabajo del estudiante, incluyendo la auto-evaluación o la revisión por una audiencia pública; y (4) la AA tiene que ofrecer más oportunidades al aprendiz para decidir en su evaluación.

Ashford-Rowe, Herrington y Brown (2014) realizan una revisión bibliográfica y establecen ocho preguntas que deben caracterizar las prácticas de AA: ¿Hasta qué punto la actividad evaluadora le supone un reto al alumno? ¿Es el proceso o el resultado el objetivo final de la evaluación? ¿Requiere la evaluación de una transferencia del aprendizaje haciendo uso de las habilidades aprendidas? ¿Requiere la evaluación de procesos metacognitivos? ¿El resultado o proceso de la evaluación puede ser reconocida como auténtica por los partícipes de la misma?

¿Son los instrumentos de evaluación reales o simulados? ¿Requiere la evaluación procesos de discusión y retroalimentación? ¿Requiere la evaluación que los alumnos colaboren entre ellos?

Murphy y otros (2017) realizan una guía con una serie de fases y pasos para que el profesorado universitario puede implementar una AA en su proceso de enseñanza, 
estableciendo cinco fases: (1) identificación de los objetivos de aprendizaje que se desean y alinearlos con las tareas que se propongan; (2) generar procesos de comunicación y consulta con los estudiantes; (3) desarrollo de rúbricas y criterios de evaluación; (4) implementación tareas de AA y mejorar el proceso aportando feedback formativo; y (5) evaluar y reflexionar sobre lo ocurrido en el proceso de evaluación.

\subsection{Experiencias y resultados de la aplicación $A A$}

Gulikers y otros (2008) realizan un estudio en el que preguntan cómo perciben los alumnos la AA y cómo condiciona esta sus aprendizajes. Sus resultados muestran que los estudiantes muy experimentados perciben mejor la influencia de la AA en el aprendizaje que los poco experimentados; sugiriendo posibles líneas de acción para desarrollar y usar la AA.

Son muchos los estudios que utilizan las características de la AA en experiencias reales. Desde el programa de doctorado de la Universidad Central de Florida llevan a cabo varios proyectos en los que utilizan una AA en los colegios y distritos de la zona; en los que los estudiantes del doctorado trabajan directamente con el colegio o distrito (Biddle ,2014). Los resultados señalan que los alumnos necesitan conocer la situación real de su futuro trabajo, conocer e interiorizar las necesidades de la organización y aprender las habilidades necesarias para desarrollar ese trabajo. Gonzalez-DeHass y Willems (2015), en unos cursos de psicología con alumnos que serán futuros profesores, desarrollan ambientes de aprendizaje auténtico y prácticas reales de cómo llevar a cabo una AA. Los resultados indican que es positivo que los maestros puedan aplicar sus aprendizajes en contextos reales y enfrentarse con experiencias reales en las que tengan que tomar sus propias decisiones.

Son varios los estudios que utilizan las nuevas tecnologías para llevar a cabo una AA. Eddy y Lawrence (2013) utilizan las Wikis como plataformas web en las que se produce a la vez aprendizaje y AA. Como resultados señalan que se producen aprendizajes auténticos y no es necesaria ninguna formación previa relacionada con la informática o la programación para realizar las actividades. Heinzen, Landrum, Gurung y Dunn (2015) consideran que llevada a cabo AA en $\mathrm{HE}$ es un reto difícil y señalan ocho grandes dificultades, utilizando la Gamificación para darles una posible solución, encontrando una motivación y actitud positiva de los estudiantes hacia este formato.

Hay varios estudios que utilizan la rúbrica y portafolio como instrumentos de evaluación imprescindibles para llevar a cabo una AA. Keyser y Howell (2008) llevan a cabo una revisión de artículos relacionados con la AA-HE, concluyendo que si los modelos teóricos que definen y describen la AA son coherentemente evaluados por rúbricas diseñadas en consonancia con los objetivos de aprendizaje, entonces se producirán no solo una evaluación eficiente, sino el desarrollo de mejores aprendizajes. Latorre y Varela (2015) señalan que las rúbricas proporcionan al estudiante una doble vía de información: le aporta una mayor información que una simple valoración de su trabajo (entendido como feedback) y le guía sobre cómo mejorar los logros alcanzados, generando un diálogo con el profesor y/o sus compañeros sobre los aspectos que debe acometer en el futuro (entendido como feedforward). Sullivan y McConnell (2017) piensan que crear rúbricas que evalúen aspectos claves del aprendizaje puede ser una herramienta útil para estructurar los tiempos en el proceso de enseñanza y cuando deben ser introducidas nuevas tareas de aprendizaje a los alumnos. Respecto al uso del portafolio, Prieto, Llacer y Escobar (2017) indican que es uno de los instrumentos más populares para pasar de una evaluación 
tradicional a otra basada en AA, ya que evalúa el desempeño de los alumnos y también mejora su aprendizaje y ayuda a que reflexionen sobre el mismo.

Otro de los aspectos tratados habitualmente es que la evaluación no sea realizada únicamente por el profesor y que puedan participar los alumnos. Kearney, Perkins y Kennedy-Clark (2016) muestran que los estudiantes son capaces de juzgar su propio trabajo y realizar evaluaciones bastante precisas del trabajo de sus compañeros, incluso aquellos que no tenían experiencia previa en autoevaluarse o en llevar a cabo una evaluación entre iguales. Sin embargo, Santos (2017) lleva a cabo una AA en la Facultad de Farmacia en donde los estudiantes encuentran difícil evaluar sus propios trabajos o los de otros compañeros al mismo nivel que lo realizan los profesores.

Febriana y Arlianty (2017) analizan en la Facultad de Química en Indonesia el posible efecto de la aplicación de AA en los futuros profesores. La herramienta de AA utilizada consistía en revisar aspectos de conocimientos, actitudes, auto-eficacia y evaluación entre iguales. Los resultados muestran mejoría en todos los aspectos señalados anteriormente. Por su parte, James y Casidy (2018) señalan que la aplicación de una AA en la Facultad de Negocios genera actitud y comportamientos positivos hacia las tareas propuestas. Por último, Nguyen (2017) lleva a cabo un estudio en un módulo sobre pedagogía en Vietnam en FIP. Para ello se implementan tres tareas de AA en la que los futuros maestros perciben que la AA influenció y cambió sus estrategias de aprendizaje y motivación, sus competencias profesionales y su identidad como profesores.

Ghosh y otros (2017) realizan una revisión de estudios AA, señalando que la tónica general es la falta de validez y fiabilidad de los mismos. Por ello, piensan que, si los aspectos de validez y fiabilidad de las prácticas AA son mejoradas desde una visión holística de la realidad, la evaluación del programa y el desarrollo de tareas por parte de los estudiantes en el lugar de trabajo pueden mejorar significativamente.

Por tanto, parecen existir orígenes diferentes del concepto "AA", desde diferentes campos profesionales y educativos. Esto hace que también podamos encontrar diferentes teorías y planteamientos sobre que es el AA y que criterios debe cumplir un sistema de evaluación para ser denominado así. A pesar de ello, si pueden encontrarse algunos criterios comunes para definir que es AA, que serán presentados en el apartado de conclusiones. La mayoría de los estudios realizados muestra resultados positivos cuando se utilizan sistemas o situaciones de AA en HE, aunque también existen trabajos que cuestionan la validez y fiabilidad de la mayoría de los estudios realizados sobre esta temática. Por otra parte, parece que los instrumentos de evaluación más adecuados y utilizados para desarrollar procesos de AA en HE son el portafolios y las rúbricas.

\section{Conclusiones}

A lo largo de este estudio hemos realizado una revisión bibliográfica en tres bases de datos internacionales de los conceptos: LOA-HE y AA-HE. Se han encontrado un total de 208 documentos de los que se han seleccionado 40. Del término LOA-HE se han encontrado 46 documentos y se han seleccionado 12, todos ellos están centrados en el ámbito de la enseñanza. Además, hay un gran número de referencias en las que Carless aparece como autor principal y/o es citado por otros autores. En cuanto al concepto de AA-HE se han encontrado 161 documentos y se han seleccionado 28 , un número considerablemente más 
alto, que muestra una mayor presencia en la literatura internacional, en parte por la mayor relación que tiene con el mundo del trabajo.

Los resultados muestran que el término LOA-HE surge inicialmente de la necesidad de responder al doble deber de la evaluación en HE: potenciar el aprendizaje del alumnado y asegurar la certificación al final de una asignatura y/o curso. Además, la LOA se intenta distanciar del término FA, ya que señalan que este último está rodeado de cierta confusión. La mayoría de los documentos señalan las características que cita Carless en sus estudios como propias de una LOA-HE: (1) las tareas de evaluación deberían ser diseñadas para estimular el aprendizaje de los alumnos; (2) la evaluación tiene que implicar a los alumnos en su propia evaluación y en la evaluación de otros compañeros; y (3) el feedback o retroalimentación ha de darse en el momento adecuado a los alumnos y que sea útil para el aprendizaje actual y futuro de los mismos. La mayoría de experiencias indican aspectos positivos en la introducción de LOA-HE en sus programas formativos en educación superior.

Por otra parte, los resultados muestran que el origen del término AA-HE se remonta al mundo del trabajo y a la necesidad de llevar a cabo en el aula tareas lo más similares posibles a las condiciones que se encontrarán los alumnos en sus futuros ambientes de trabajo. Sin embargo, hay otros documentos que señalan las virtudes de poder llevar a cabo una AA con una finalidad educativa, en la que los dos grandes instrumentos de evaluación son el diseño de rúbricas y el portafolio. Además, un gran número de documentos señalan unos criterios comunes para llevar a cabo una AA: (1) llevar a cabo tareas auténticas en el aula, con características similares o iguales a las de la vida real; y (2) contexto similar o mimetizado al que se encontrarán en el trabajo. La mayoría de experiencias indican que los alumnos que reciben este tipo de evaluación la valoran muy positivamente, especialmente la conexión con su futuro laboral, las habilidades requeridas y la puesta en práctica en situaciones reales.

Este estudio es clave en el estado actual de la evaluación en el EEES. La mayoría de revisiones bibliográficas relacionadas con FA-HE están hechas en inglés, poco actualizadas y sin considerar los conceptos de LOA-HE y AA-HE, que son muy utilizados actualmente en la literatura internacional. Por ello, este trabajo presenta una clarificación terminológica en castellano y las características básicas de cada concepto, que son congruentes con las exigencias que implica el EEES. Por todo ello puede resultar un trabajo muy útil para todo el profesorado universitario interesado en estas temáticas, así como los gabinetes de evaluación y mejora de las universidades iberoamericanas.

Como prospectiva, sería interesante investigar si se están publicando en español experiencias y estudios relacionadas con LOA-HE y AA-HE y que resultados están obteniendo; así como la posible comparativa de estos resultados con los encontrados a nivel internacional.

\section{Referencias}

Archbald, D. A. (1991). Authentic assessment: principles, practices, and issues. School Psychology Quarterly, 6(4), 279-293. https://doi.org/10.1037/hoo88821

Archbald, D. A. y Newmann, F. M. (1988). Assessing authentic academic achievement in the secondary school. NASSP. 
Ashford-Rowe, K., Herrington, J. y Brown, C. (2014). Establishing the critical elements that determine authentic assessment. Assessment Eं Evaluation in Higher Education, 39(2), 205222. https://doi.org/10.1080/02602938.2013.819566

Barrientos, E. (2013). La evaluación formativa y evaluación orientada al aprendizaje en educación superior: una revisión internacional. Universidad de Valladolid.

Biddle, J. (2014). A needs analysis for K-12 school improvement projects and their use as the dissertation in practice for the professional practice education doctorate program at the University of Central Florida. University of Central Florida.

Black, P. y Wiliam, D. (1998). Assessment and classroom learning. Assessment in Education: Principles, Policy E̊ Practice, 5(1), 7-74. https://doi.org/10.1080/0969595980050102

Bohemia, E. y Davison, G. (2012). Authentic learning: the gift project. Design and Technology Education: an International Journal, 17(2), 49-61.

Bosco, A. M. y Ferns, S. (2014). Embedding of authentic assessment in work-integrated learning curriculum. Asia-Pacific Journal of Cooperative Education, 15(4), 281-290.

Boud, D. y Falchikov, N. (2006). Aligning assessment with long-term learning. Assessment y Evaluation in Higher Education, 25(3), 279-291. https://doi.org/10.1080/02602930600679050

Bretones, A. (2008). Participación del alumnado de Educación Superior en su evaluación. Revista de Educación, 347, 181-202.

Canabal, C. y Margalef, L. (2017). The feedback: a key to learning-oriented assessment. Profesorado. Revista de Curriculum y Formación de Profesorado, 21(2), 149-170.

Carless, D. (2007). Learning-oriented assessment: conceptual bases and practical implications. Innovations in Education and Teaching International, 44(1), 57-66. https://doi.org/10.1080/14703290601081332

Carless, D. (2009a). Learning-oriented assessment: principles, practice and a project. En L. H. Meyer, S. Davidson, H. Anderson, R. Fletcher, P. M. Johnston y M. Rees (Eds.), Tertiary assessment y higher education student outcomes: Police, practice $\sigma^{\circ}$ research learning-oriented assessment (pp. 79-90). Ako Aotearoa.

Carless, D. (2009b). Trust, distrust and their impact on assessment reform. Assessment \&ٔ Evaluation in Higher Education, 34(1), 79-89. https://doi.org/10.1080/02602930801895786

Carless, D. (2015a). Excellence in university assessment: learning from award-winning practice. Routledge.

Carless, D. (2015b). Exploring learning-oriented assessment processes. Higher Education, 69(6), 963-976. https://doi.org/10.1007/s10734-014-9816-Z

Carless, D., Joughin, G. y Mok, M. (2006). Learning-oriented assessment: principles and practice. Assessment \& Evaluation in Higher Education, 31(4), 395-398. https://doi.org/10.1080/02602930600679043

Carmona, M. y Flores, J. (2008). Learning-oriented assessment in higher education: conditions and strategies for its application to university teaching. Revista Española de Pedagogía, 241, 467486.

Cumming, J. y Maxwell, G. S. (1999). Contextualising authentic assessment. Assessment in Education: Principles, Policy \& Practice, 6(2), 177-194.

https://doi.org/10.1080/09695949992865 
Dochy, F., Segers, M. y Dierick, S. (2002). Nuevas vías de aprendizaje y enseñanza y sus consecuencias: una nueva era de evaluación. Revista de Docencia Universitaria, 2(2), 13-29.

Eddy, P. L. y Lawrence, A. (2013). Wikis as platforms for authentic assessment. Innovative Higher Education, 38(4), 253-265. https://doi.org/10.1007/s 10755-012-9239-7.

Febriana, B. y Arlianty, W. (2017) The application of authentic assessment in chemistry curriculum. En B. Budi, L. Kim, Y. Isao e I. Khan (Eds.), Proceedings of the 3rd international xonference on education and training (pp. 99-105). International Institute of Knowledge Management. https://doi.org/10.17501/icedu.2017.3111

Gauntlett, N. (2007). Literature review on formative assessment in higher education. Middlesex University.

Gessa Perera, A. (2011). La coevaluación como metodología complementaria de la evaluación del aprendizaje. Análisis y reflexión en las aulas universitarias. Revista de Educación, 354, 749764 .

Ghosh, S., Bowles, M., Ranmuthugala, D. y Brooks, B. (2017). Improving the validity and reliability of authentic assessment in seafarer education and training: a conceptual and practical framework to enhance resulting assessment outcomes. WMU Journal of Maritime Affairs, 16(3), 455-472. https://doi.org/10.1007/s 13437-017-0129-9

Gonzalez-DeHass, A. R. y Willems, P. P. (2015). Case-study instruction in educational psychology: implications for Ttacher preparation. En M. Li y Y. Zhao (Ed.), Exploring learning Ẽ teaching in higher education (pp. 99-122). Springer.

Gulikers, J. M., Bastiaens, T. J. y Kirshner, P. A. (2004). A five-dimensional framework for authentic assessment. Educational Technology Research and Development, 52(3), 67-87. https://doi.org/10.1007/BFo2504676

Gulikers, J. T., Kester, L., Kirschner, P. A. y Bastiaens, T. J. (2008). The effect of practical experience on perceptions of assessment authenticity, study approach, and learning outcomes. Learning and Instruction, 18(2), 172-186.

https://doi:10.1016/j.learninstruc.2007.02.012

Hassanpour, B., Utaberta, N., Abdullah, N. y Tahir, M. (2011). Authentic assessments or standardized assessment new attitude to architecture assessment. Procedia-Social and Behavioral Sciences, 15, 3590-3595. https://doi.org/10.1016/j.sbspro.2011.04.340

Heinzen, T. E., Landrum, R. E., Gurung, R. A. y Dunn, D. S. (2015). Game-based assessment: the mash-up we've been waiting for. En T. Reiners y L. Wood (Eds.), Gamification in education and business (pp. 201-217). Springer.

Ibarra, M. S., Rodríguez, G. y Gómez, M. A. (2012). La evaluación entre iguales: beneficios y estrategias para su práctica en la universidad. Revista de Educación, 359, 206-231. https://doi.org/10-4438/1988-592X-RE-2010-359-092

James, L. T. y Casidy, R. (2018). Authentic assessment in business education: its effects on student satisfaction and promoting behaviour. Studies in Higher Education, 43(3), 401-415. https://doi.org/10.1080/03075079.2016.1165659

Kaider, F., Hains-Wesson, R. y Young, K. (2017). Practical typology of authentic work-integrated learning activities and assessments. Asia-Pacific Journal of Cooperative Education, 18(2), 153165.

Kearney, S., Perkins, T. y Kennedy-Clark, S. (2016). Using self-and peer-assessments for summative purposes: analysing the relative validity of the AASL (Authentic Assessment for Sustainable Learning) model. Assessment E Evaluation in Higher Education, 41(6), 840-853. https://doi.org/10.1080/02602938.2015.1039484 
Keppell, M. y Carless, D. (2006). Learning-oriented assessment: a technology-based case study. Assessment in Education: Principles, Policy E Practice, 13(2), 179-191. https://doi.org/10.1080/09695940600703944

Keyser, S. y Howell, S. L. (2008). The state of authentic assessment. Columbus.

Latorre, M. A. y Varela, J. L. M. (2015). La contribución de las rúbricas a la práctica de la evaluación auténtica. Observar, 9, 5-17.

Lombard, B. (2008). Modelling critical thinking through learning-oriented assessment. South African Journal of Higher Education, 22(5), 1029-1043. https://doi.org/10.4314/sajhe.v22i5.42923

López-Pastor, V. M. (2008). Implementing a formative and shared assessment system in higher education teaching. European Journal of Teacher Education, 31(3), 293-311. https://doi.org/10.1080/02619760802208452

López-Pastor, V. M., D. Kirk, E. Lorente-Catalán, MacPhail, A. y Macdonald, D. (2013). Alternative assessment in physical education: a review of international literature. Sport, Education E Society, 18(1), 57-76. https://doi.org/10.1080/13573322.2012.713860

López-Pastor, V. y Sicilia-Camacho, A. (2017). Formative and shared assessment in higher education. Lessons learned and challenges for the future. Assessment \& Evaluation in Higher Education, 42(1), 77-97. https://doi.org/10.1080/02602938.2015.1083535

Lorente, E. y Kirk, D. (2013). Alternative democratic assessment in PETE: an action-research study exploring risks, challenges and solutions. Sport, Education and Society, 18(1), 77-96. https://doi.org/10.1080/13573322.2012.713859

McDermott, M. Z. R., Daniels, M., Nylén, A., Pears, A., Isomöttönen, V. y Caspersen, M. (2017). The authenticity of 'authentic'assessment some faculty perceptions. En M. E. Van Valkenburg (Ed.), Proceedings of IEEE frontiers in education conference (pp. 1-9). IEEE.

Murphy, V., Fox, J., Freeman, S. y Hughes, N. (2017). “Keeping it Real”: A review of the benefits, challenges and steps towards implementing authentic assessment. AISHE-J: The All Ireland Journal of Teaching and Learning in Higher Education, 9(3).

Nguyen, H. (2017). Authentic assessment in pedagogy-related modules in teacher education: Vietnamese student teachers' perspective. University of East Anglia.

Palacios, A. y López-Pastor, V. M. (2013). Haz lo que yo digo pero no lo que yo hago: sistemas de evaluación del alumnado en la formación inicial del profesorado. Revista de Educación, 361, 279-305. https://doi.org/10.4438/1988-592X-RE-2011-361-143.

Pérez, A., Tabernero B., López Pastor, V. M., Ureña, N., Ruiz Lara, E., Caplloch, M., González Fernández, N. y Castejón, F. J. (2008). Evaluación formativa y compartida en la docencia universitaria y el Espacio Europeo de Educación Superior: cuestiones clave para su puesta en práctica. Revista de Educación, 347, 435-451.

Prieto, J. P. A., Llácer, C. V. H. y Escobar, A. H. (2017). The effect of portfolios on higher Education students learning. En L. Gómez Chova, A. López Martínez, I. Candel Torres (Eds.), Proceedings of 11 th international technology, education and development conference (pp. 64786480). IATED Academy.

Quesada, V., Rodríguez, G. e Ibarra, M. S. (2013). ActEval: un instrumento para el análisis y la reflexión sobre la actividad evaluadora del profesorado universitario. Revista de Educación, 362, 69-104. https://doi.org/10.4438/1988-592X-RE-2011-362-153

Rodríguez, G., Quesada, V. e Ibarra, M. (2016). Learning-oriented e-assessment: the effects of a training and guidance programme on lecturers' perceptions. Assessment E Evaluation in Higher Education, 41(1), 35-52. https://doi.org/10.1080/02602938.2014.979132 
Rule, A. C. (2006). The components of authentic learning. The Journal of Authentic Learning, 3(1), $1-10$.

Santos, J. M. (2017). Design, implementation and evaluation of an authentic assessment experience in a pharmacy course: are students getting it? En J. Domenech, M. C. Vincent Vela, E. Poza Plaza y M. D. Blázquez Soriano (Eds), Proceedings of the 3rd international conference on higher education advances (pp. 574-583). Universitat Politécnica de Valencia. https://doi.org/10.4995/HEAd 17.2017.5294

Sullivan, D. F. y McConnell, K. D. (2017). Big progress in authentic assessment, but by itself not enough. Change: The Magazine of Higher Learning, 49(1),14-25.

https://doi.org/10.4995/HEAD17.2017.5294

Vu, T. T. (2011). Investigating authentic assessment for student learning in higher education. University of Queensland.

Zabalza, M. Á. (2003). Competencias docentes del profesorado universitario. Calidad y desarrollo profesional. Narcea.

\section{Breve $\mathrm{Cv}$ de los autores}

\section{Emilio José Barrientos-Hernán}

Doctor en Educación y Licenciado en Psicopedagogia. Maestro de Educación Primaria especialidad en Educación Física, con 10 años de experiencia en Educación Primaria. ORCID ID: https://orcid.org/OOOO-0002-6060-8486. Email:

barrientosemilio@gmail.com

\section{Víctor M. López-Pastor}

Profesor de la Facultad de Educación de Segovia (Universidad de Valladolid). Catedrático de Universidad. Ha publicado 30 libros y numerosos artículos científicos y profesionales. Sus principales líneas de investigación son: *Evaluación Formativa en educación superior, *Evaluación Formativa en educación física, *Formación del Profesorado, *InvestigaciónAcción. ORCID ID: https://orcid.org/o000-0003-2681-9543. Email:vlopez@mpc.uva.es

\section{Dario Pérez-Brunicardi}

Doctor en Educación (UVa) y Licenciado en Educación Física (UMP). Profesor Contratado Doctor de la Facultad de Educación de Segovia. Coordinador de la Red Estatal de Educación Física en la Naturaleza. Líneas de Investigación: Educación Física en la Naturaleza, Expresión Corporal, Deporte Escolar y Evaluación para el Aprendizaje. ORCID ID: https://orcid.org/OOOO-OOO2-1347-1333. Email:

dario.perez.brunicardi@gmail.com 